\title{
Powerful photomixers
}

\author{
An efficient continuous-wave source of terahertz radiation that combines the outputs from two near-infrared \\ semiconductor lasers in a novel photomixer looks set to benefit applications in spectroscopy and imaging.
}

\section{Tahsin Akalin}

T erahertz (THz) waves, which lie between the microwave and optical regions of the electromagnetic spectrum, have become a valuable resource for spectroscopy and imaging, enabling many useful tasks in the fields of security screening, biomedical imaging, chemical analysis and high-speed wireless communication. For example, $\mathrm{THz}$ instruments can be used to detect explosives, skin cancer and metal objects hidden beneath clothing, as well as perform a variety of other tasks.

Unfortunately, however, the cost and availability of $\mathrm{THz}$ equipment has so far been hindered by the lack of compact, powerful and convenient sources of $\mathrm{THz}$ waves that operate at room temperature. Now, writing in Nature Photonics, Hendrix Tanoto and co-workers from A*STAR and the National University of Singapore and Imperial College London report a promising solution to this problem ${ }^{1}$.

Their approach involves mixing the light from two tunable distributed feedback lasers, which operate around a wavelength of $852 \mathrm{~nm}$, in a novel semiconductor photomixer made from a GaAs photoconductive antenna. The resulting beat-frequency - the difference between the emission frequencies of the two lasers - is generated in the $\mathrm{THz}$ domain and then outcoupled and radiated by the antenna. The photomixer employs tip-to-tip nanoelectrodes in the shape of a nanostructured metal comb in the semiconductor active region (Fig. 1) and a well-matched antenna that serves to increase the mixing process and emitted $\mathrm{THz}$ power by two orders of magnitude over conventional designs. This remarkable performance improvement, reaching emission powers in the tens and hundreds of microwatts, represents a significant breakthrough for $\mathrm{THz}$ generation using a photoconductive antenna.

Simulations by Tanoto et al. show that the nanogap of the tip-to-tip electrode structure can enhance the field strength of a $1 \mathrm{THz}$ wave by as much as a factor of 400 for a $50 \mathrm{~nm}$ gap and 100 for a $100 \mathrm{~nm}$ gap, when the field is aligned

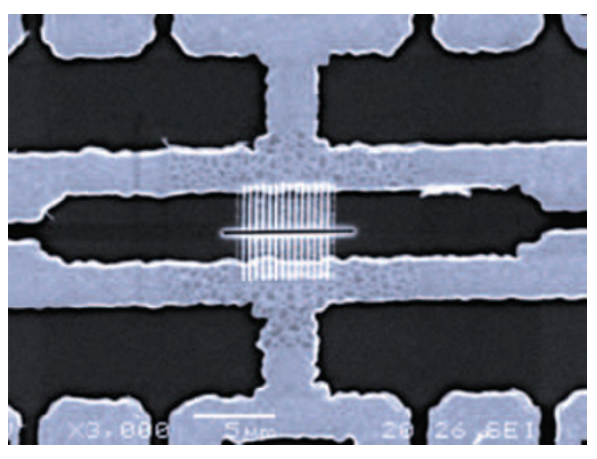

Figure1 | The tip-to-tip nanogap electrode structure employed by Tanoto et al. in their semiconductor photoconductive antenna. The tiny gap dramatically enhances the strength of the $\mathrm{THz}$ field while also actively improving the signal.

along the direction of the electrode fingers. This improvement is achieved at room temperature, which makes the scheme particularly beneficial for general-purpose applications.

$\mathrm{THz}$ signals can currently be generated through one of three distinct techniques. The first is the upconversion of microwave signals to the $\mathrm{THz}$ domain. The second is the downconversion of optical or infrared signals to $\mathrm{THz}$ waves using a photomixer. The third is the use of optoelectronic devices - in particular quantum cascade lasers - to generate signals directly in the $\mathrm{THz}$ band. For descriptions of these different techniques, the reader may wish to refer to a range of informative work on the subject ${ }^{2-4}$.

Fortunately, up- and downconversion techniques can exploit the wide availability of powerful sources developed for use at microwave and optical frequencies, where commercial applications such as telecommunications have driven considerable investment in source technology.

One of the issues that must be tackled when designing a $\mathrm{THz}$ source is how to achieve efficient outcoupling of the generated $\mathrm{THz}$ power, particularly if the source is to be used in a small electronic device. Several techniques have been developed to this end, including $\mathrm{THz}$ quantum cascade lasers that use plasmonic arrays $^{5}$ to improve the radiation beam using constructive interference and thus increase the total radiated power. Another approach that utilizes plasmonic effects is the exploitation of semiconductor resonances to improve the source outcoupling ${ }^{6}$.

It is clear that the use of plasmonics and metamaterials in the $\mathrm{THz}$ domain is growing in popularity and represents a promising emerging field of research for designing next-generation $\mathrm{THz}$ devices ${ }^{7-9}$. Several research groups have been working on increasing the conversion efficiency of $\mathrm{THz}$ sources, which is one of the most important parameters for improving the total generated power output. The concepts of SPASERs (surface plasmon amplification by the stimulated emission of radiation) and SPIDERs (surface-plasmon-induced drag effect) will also play a role in the development of new plasmonic devices ${ }^{10-11}$.

In the future, it is likely that nanostructured engineering will be increasingly used to enhance the performance of $\mathrm{THz}$ sources and other $\mathrm{THz}$ devices. Indeed, the latest results of Tanoto et al. are an excellent example of what can be achieved and are a cause for optimism. These devices will hopefully find their way into applications such as $\mathrm{THz}$ wireless communications, imaging techniques and near-field microscopy.

Tahsin Akalin is Associate Professor in the Terahertz Photonics Group at Lille 1 University (IEMN/IUT A), Villeneuve d'Ascq, France. e-mail:Tahsin.Akalin@iemn.univ-lille1.fr

\footnotetext{
References

1. Tanoto, H. et al. Nature Photon. 6, 121-126 (2012).

2. Tonouchi, M. Nature Photon. 1, 97-105 (2007).

3. Preu, S., Döhler, G. H., Malzer, S., Wang, L. J. \& Gossard, A. C. J. Appl. Phys. 109, 061301 (2011).

4. Williams, B. Nature Photon. 1, 517-525 (2007).

5. Nanfang, Y. et al. Nature Mater. 9, 730-735 (2010).

6. Giannini, V., Berrier, A., Maier, S. A., Sánchez-Gil, J. A. \& Rivas, J. G. Opt. Express 18, 2797-2807 (2010).

7. Navarro-Cía, M., Beruete, M., Sorolla, M. \& Campillo, I. Opt. Express 16, 560-566 (2008).

8. Zhu, W., Agrawal, A., Cui, A., Kumar, G. \& Nahata, A. IEEE J. Sel. Top. Quant. Electron. 17, 146-153 (2011).

9. Chen, W.-C., Mock, J. J., Smith, D. R., Akalin, T. \& Padilla, W. J. Phys. Rev. X 1, 021016 (2011)

10. Stockman, M. I. Phys. Rev. Lett. 106, 156802 (2011).

11. Durach, M. I., Rusina, A. \& Stockman, M. I. Phys. Rev. Lett. 103, 186801 (2009).
} 